\title{
The Small Ones Matter-sHsps in the Bacterial Chaperone Network
}

\author{
Igor Obuchowski*, Piotr Karaś and Krzysztof Liberek* \\ Intercollegiate Faculty of Biotechnology UG-MUG, University of Gdansk, Gdansk, Poland
}

Small heat shock proteins (sHsps) are an evolutionarily conserved class of ATPindependent chaperones that form the first line of defence during proteotoxic stress. sHsps are defined not only by their relatively low molecular weight, but also by the presence of a conserved $\alpha$-crystallin domain, which is flanked by less conserved, mostly unstructured, $\mathrm{N}$ - and C-terminal domains. sHsps form oligomers of different sizes which deoligomerize upon stress conditions into smaller active forms. Activated sHsps bind to aggregation-prone protein substrates to form assemblies that keep substrates from irreversible aggregation. Formation of these assemblies facilitates subsequent Hsp70 and Hsp100 chaperone-dependent disaggregation and substrate refolding into native

OPEN ACCESS

Edited by:

Axel Mogk,

Heidelberg University, Germany

Reviewed by:

Martin Haslbeck,

Technical University of Munich,

Germany

Elizabeth Vierling,

University of Massachusetts Amherst,

United States

${ }^{*}$ Correspondence:

Krzysztof Liberek

krzysztof.liberek@ug.edu.pl

Igor Obuchowski

igor.obuchowski@gmail.com

Specialty section:

This article was submitted to

Protein Folding, Misfolding and

Degradation,

a section of the journal

Frontiers in Molecular Biosciences

Received: 11 February 2021

Accepted: 28 April 2021

Published: 13 May 2021

Citation:

Obuchowski I, Karaś $P$ and Liberek K (2021) The Small Ones Matter-sHsps

in the Bacterial Chaperone Network.

Front. Mol. Biosci. 8:666893.

doi: 10.3389/fmolb.2021.666893 species. This mini review discusses what is known about the role and place of bacterial sHsps in the chaperone network.

Keywords: protein aggregation, protein refolding, holdase activity, chaperones, small heat shock proteins (sHsps), proteotoxic stress, Hsp70-Hsp100 dependent disaggregation

\section{INTRODUCTION}

Bacterial sHsps, unlike most other chaperones, were discovered later than their eukaryotic homologues. They were originally found in Escherichia coli inclusion bodies (Allen et al., 1992), hence they were given names IbpA and IbpB inclusion body-associated protein A and B. They were later reported to interact with endogenous polypeptides upon heat stress conditions and therefore classified as members of the chaperone family (Laskowska et al., 1996).

The level of sHsps in bacteria is very low at physiological conditions. This is due to very tight regulation of sHsp expression at both transcriptional and translational levels. In E. coli ibpA and ibpB genes are arranged into an operon which is controlled by $\sigma^{32}$, the main heat shock response regulator (Allen et al., 1992; Chuang and Blattner 1993; Kuczyńska-Wisńik et al., 2001). The deletion of the $i b p A B$ operon does not influence $E$. coli growth in permissive conditions, however during prolonged harsh stress it substantially decreases bacterial viability (Kuczynska-Wisnik et al., 2002).

After transcription at a permissive temperature, the $i b p A B$ mRNA forms a hairpin structure, which restricts access to its own Shine-Dalgarno sequence (SD) (Waldminghaus et al., 2009; Gaubig et al., 2011), preventing unnecessary translation. Additionally, oligomeric IbpA negatively regulates its own translation by directly binding to $i b p A B$ mRNA, which promotes the mRNA degradation by polynucleotide phosphorylase (Miwa et al., 2021). At the protein level, excessive sHsps are effectively degraded by Lon protease (Bissonnette et al., 2010).

At stress conditions the expression of sHsps rapidly increases. This is orchestrated by the $\sigma^{32}$ transcription activation, meltdown of the SD-covering mRNA hairpin structure (Waldminghaus et al., 2009) and heat-induced deoligomerization and dissociation of IbpA from its own mRNA (no more degradation stimulation) (Miwa et al., 2021). This, in E. coli, causes $~ 300$ fold induction of the sHsp expression at the transcriptional level (Richmond et al., 1999), which results in a very dynamic 20 -fold increase in the cellular abundance of sHsps (Valdez-Cruz et al., 2011; Laskowska et al., 1996; 
Mogk et al., 1999). This is in contrast to other heat shock proteins, whose cellular levels typically increase only 2-3 times in similar conditions (Mogk et al., 1999).

Such unusually tight multilevel control of IbpA and IbpB expression in $E$. coli points to their importance at stress conditions and suggests that at physiological conditions sHsps may exert some negative effects on bacterial growth. Indeed, it was recently observed that the overexpression of IbpA inhibits E. coli growth (Miwa et al., 2021). It was also observed that the expression of Mycobacterium tuberculosis sHsp16.3 arrests cell growth, which in the case of TB is beneficial, as it allows the bacteria to establish the characteristic latent infection ( $\mathrm{Hu}$ et al., 2006).

Although bacterial sHsp expression studies explored mostly E. coli, less investigated bacterial systems seem to generally show similar trends of the heat-dependent sHsps expression. Analyzing sHsp genes from multiple alpha- and gamma-proteobacteria, Narberhaus and colleagues have shown that, similarly to E. coli sHsps, they possess RNA thermometers within SD sequences (Narberhaus et al., 2006) that form hairpins on the mRNA structure and melt upon a temperature rise to promote the translation initiation.

\section{STRUCTURE OF BACTERIAL SMALL HEAT SHOCK PROTEINS}

The secondary and tertiary structure of bacterial sHsps is highly conserved. The central $\sim 90$ aa $\alpha$-crystallin domain is the basic structural element which defines the membership in the sHsp family (Haslbeck and Vierling 2015; Basha et al., 2013). The a-crystallin domain consists of two antiparallel $B$-sheets, formed by three and four $\beta$-strands, as well as an extended, so-called dimerization loop. This structure is conserved among bacterial and other, non-metazoan sHsps (Hilario et al., 2011; Mani et al., 2016). The a-crystallin domain is flanked by highly divergent, partially unstructured, flexible $\mathrm{N}$ - and $\mathrm{C}$ - terminal extensions. These tend to be enriched in prolines, which may contribute to the reduced amount of secondary structures present in these termini (Kriehuber et al., 2010). A highly conserved feature of the C-terminal extension is the (I/V)-X-(I/V) motif (Haslbeck and Vierling 2015), preceded by a positively charged amino acid (in E. coli IbpA - arginine 133) (Strozecka et al., 2012).

A characteristic feature of all sHsps is their ability to form oligomers. Known structures of bacterial sHsp oligomers include tetrahedral 12-mers formed by $M$. tuberculosis Hsp 16.3 (Kennaway et al., 2005), as well as the 18-meric trigonal bipyramid and the 24-meric octahedron formed by Salmonella typhimurium AgsA (Mani et al., 2016). Deinococcus radiodurans Hsp 20.2 is able to form 18-mers and 36-mers (Bepperling et al., 2012) and E. coli IbpA and IbpB form large, polydisperse oligomers up to several $\mathrm{MDa}$ in size (Shearstone and Baneyx 1999; Matuszewska et al., 2005), IbpA also being able to form fibrils in vitro in the absence of IbpB (Ratajczak et al., 2010).

The oligomers are formed by sHsp dimers that interact with each other and build higher-order structures (Kennaway et al.,
2005; Hilario et al., 2011; Mani et al., 2016). A notable exception is Hsp 17.7 from D. radiodurans that does not form higher-order oligomers and exists exclusively as a dimer (Bepperling et al., 2012). Interactions between $\boldsymbol{\alpha}$-crystallin domains play a crucial role in the formation of the dimers. In the case of bacterial sHsps, the dimer is stabilized mainly by interactions between the extended loop on one monomer and two $\beta$ - strands on the other monomer (Hilario et al., 2011; Bepperling et al., 2012; Mani et al., 2016).

While not required for sHsp dimerization, the $\mathrm{N}$ - and $\mathrm{C}$ terminal extensions play a crucial role in the formation of higherorder oligomers (Mani et al., 2016; Strozecka et al., 2012; Bepperling et al., 2012; Fu et al., 2005). The conserved C-terminal (I/V)-X-(I/V) motif interacts with a hydrophobic groove formed by two $\beta$-sheets on the $\alpha$-crystallin domain of the other sHsp, providing an anchoring interaction between adjacent dimeric units in the sHsp oligomer (Kennaway et al., 2005; Bepperling et al., 2012). The N-terminal extensions tend to group together inside the oligomer structure and their deletion prevents the formation of higher order oligomers (Kennaway et al., 2005). Current understanding of the N-terminal extension detailed role in bacterial sHsp oligomerization is limited by the difficulty in obtaining high-quality crystallographic data, likely due to N-terminus mobility in the oligomer (Hilario et al., 2011; Mani et al., 2016).

\section{ACTIVITIES OF BACTERIAL SMALL HEAT SHOCK PROTEINS}

Since eukaryotic (also human) sHsps were discovered before their bacterial homologues, the majority of biochemical data describing sHsp activities come from eukaryotic systems studies. It is somehow anticipated that bacterial sHsps possess similar biochemical properties since sHsps from both groups are structurally similar (Haslbeck et al., 2019). Both eukaryotic (Friedrich et al., 2004; Painter et al., 2008; Benesch et al., 2010) and bacterial sHsps oligomer populations (Shearstone and Baneyx 1999; Jiao et al., 2005) are in dynamic equilibrium and upon temperature raise tend to shift toward smaller species. Oligomerized sHsps are considered an inactive, storage form of sHsps and it is the heat-dissociated smaller species (dimers?), that are believed to be responsible for their chaperone activity (Haslbeck and Vierling 2015).

The canonical chaperone activity of sHsps is ATPindependent and is based on scavenging unfolding polypeptides before they spontaneously aggregate and either quickly releasing them after a swift stabilization or more permanently complexing them into so-called sHsp-substrate assemblies (Figure 1) - at least in vitro (Haslbeck et al., 2005). It is still unclear what is the discriminating factor that drives the process towards either the first or the second path, it is however speculated to rely on the unfolding state/hydrophobicity of the substrate.

The quick bind-and-release activity path of bacterial sHsps can be observed both in vitro and in vivo in enzyme activity 


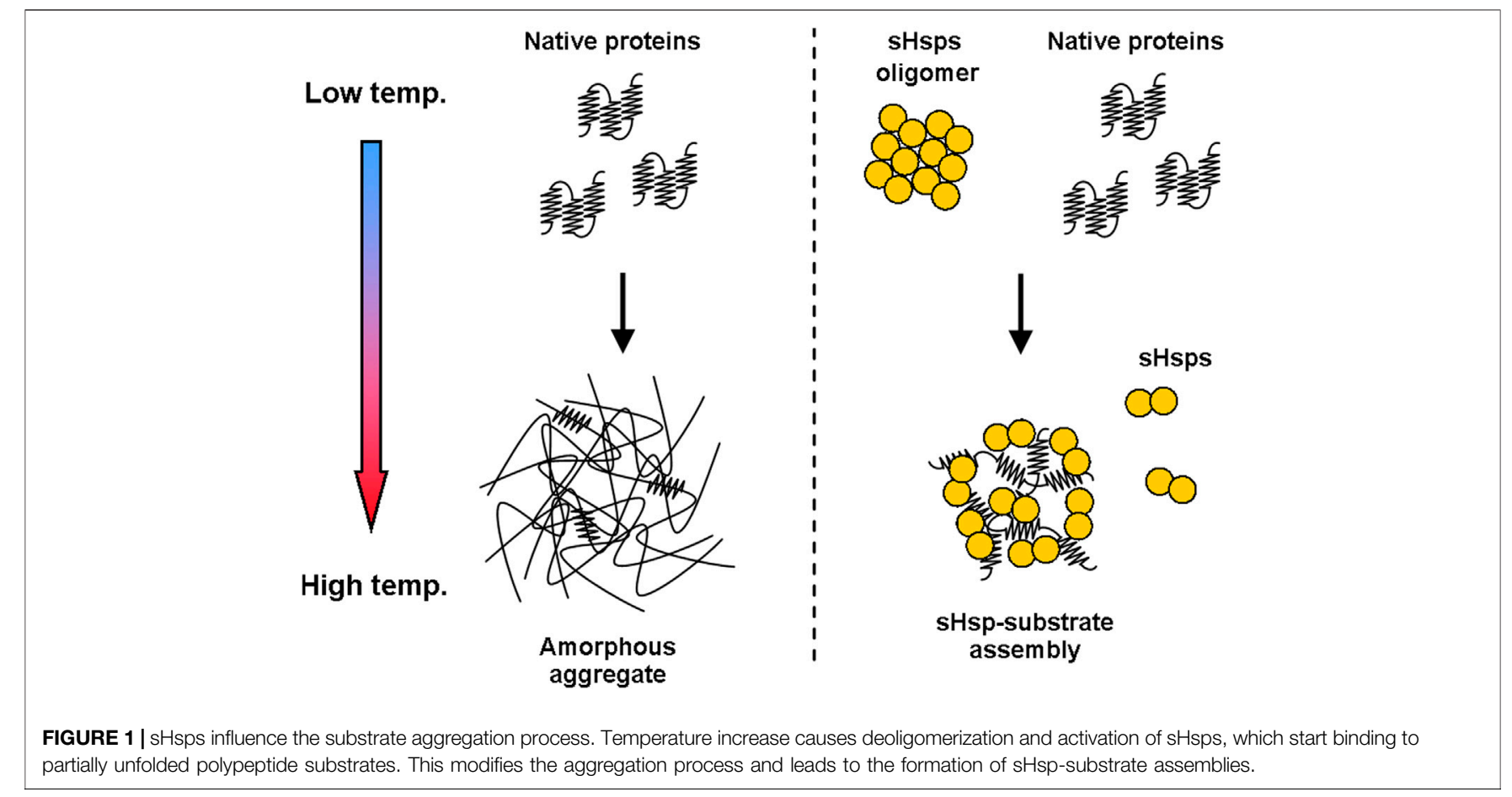

protection assays. In this way E. coli sHsps were shown to protect different enzymes from thermal (Fu et al., 2013; Matuszewska et al., 2005), oxidative and freeze-thaw (Kitagawa et al., 2002) inactivation. On the other hand, however, there are sHsps that are completely ineffective in this mode of activity. In turn, they are capable of stably binding polypeptides and driving their aggregation towards small assemblies (Chang et al., 1996). In fact, there are species like $D$. radiodurans that possess two different non-interacting sHsps, where each seems to be dedicated to either transient or stable interactions with unfolding polypeptides (Bepperling et al., 2012). This is in contrast to E. coli, where both expressed sHsps can, to some extent, protect enzymes from inactivation (Kitagawa et al., 2002; Matuszewska et al., 2005) and cooperate in stable substrate binding and disaggregation (Matuszewska et al., 2005; Ratajczak et al., 2009; Zwirowski et al., 2017). Therefore, it is not only substrate hydrophobicity but also an inherent property of the sHsp that decides whether to bind stably or transiently.

Bacterial sHsps, similarly to their eukaryotic homologues, are considered to bind aggregation-prone polypeptides via the $\mathrm{N}$-terminus, which is uncovered by a thermal dissociation of sHsp oligomers (Strozecka et al., 2012; Altenhoff et al., 2013; Chernova et al., 2020), and hydrophobic patches of the a-crystallin domain (Fu et al., 2013). Intermediate sHsppolypeptide complexes may later associate into bigger assemblies comprised of both unfolded substrates and multiple sHsps. These constitute a safe-storage for clusters of folding intermediates that are protected from further aggregation by an sHsp outer shell (Zwirowski et al., 2017). sHsps interaction with unfolding substrate not only protects the substrate from further aggregation but also preserves the substrate secondary structure (Ungelenk et al., 2016). However, it was only shown using yeast sHsps and the analogous activity for bacterial sHsps has to be confirmed.

In vitro work has revealed that assemblies built of sHsps and substrates are substantially smaller than substrate amorphous aggregates formed in the same conditions in the absence of sHsps (Chang et al., 1996; Ratajczak et al., 2009; Obuchowski et al., 2019). As a consequence, the surface to mass ratio for the assemblies is much bigger, which generates more sites at which the disaggregation and substrate refolding may potentially start. However, it is not known if in vivo association of sHsps with denatured substrates increases the surface to mass ratio, as observed in vitro.

In addition to the classical chaperone activity towards proteins, some sHsps were found to participate in membrane maintenance in Synechocystis PCC 6803 and Oenococcus oeni (Horvath et al., 1998; Torok et al., 2001; Maitre et al., 2014). Analogically, this activity is exerted by dissociated species that bind to the bacterial inner membrane, reducing its fluidity in stress conditions or in the presence of organic solvents (Torok et al., 2001; Capozzi et al., 2011; Maitre et al., 2012). sHsps were also found to stabilize thylakoid membranes in photosynthesizing cyanobacteria (Nakamoto and Honma 2006) or be involved in membrane fluidification in Lactobacillus plantarum, contributing to its cryotolerance (Arena et al., 2019). Despite the substrate difference, these activities seem similar to the classical chaperone protective activity and therefore the proteins exerting such activities (sHsps) were named lipochaperones (Maitre et al., 2014). 

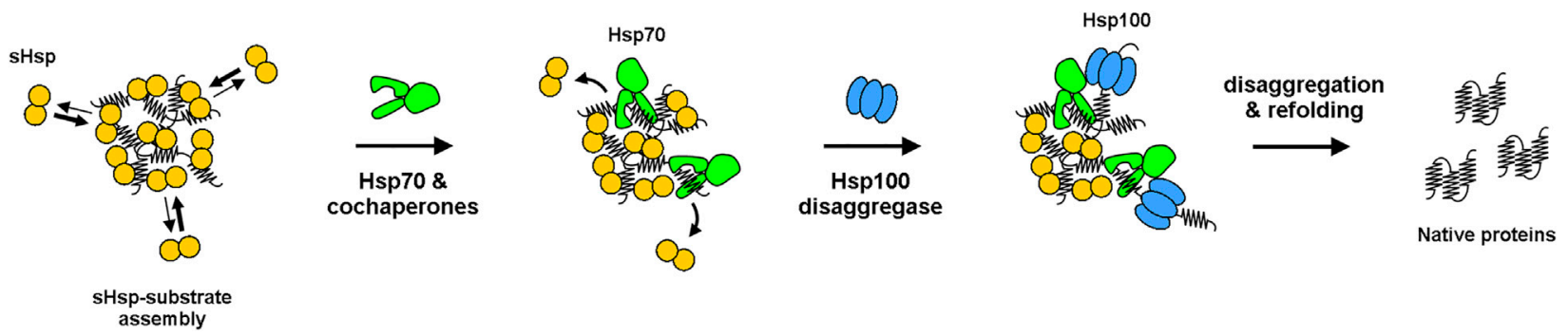

FIGURE 2 | Model of refolding of substrates from sHsp-substrate assemblies by Hsp100-Hsp70 bi-chaperone system. sHsps dynamically bind and dissociate from the assemblies, competing with Hsp70 for binding sites. Binding of Hsp70 to assemblies allows for Hsp100 (ClpB) disaggregase recruitment, which initiates substrate disaggregation and refolding.

\section{SMALL HEAT SHOCK PROTEINS COOPERATE FUNCTIONALLY WITH HSP70 AND HSP100 CHAPERONES IN REFOLDING OF THE AGGREGATED SUBSTRATE}

The introduction of sHsps to the family of molecular chaperones has raised fundamental questions regarding their possible relations to other, ATP-dependent chaperones in orchestrating cellular proteostasis. This drove the research on sHsps toward more precise integration in the network of molecular chaperones and their interactions. As described in the previous section, sHsps were shown to create sHsps-substrate assemblies upon aggregation initiation, which provoked the obvious concerns about the later fate of these structures.

There are two possible scenarios for protein aggregates-either degradation by proteases or disaggregation and refolding. The second is mediated by Hsp70 system (DnaK, DnaJ and GrpE cochaperones in bacteria) either cooperating with Hsp100 disaggregase ( $\mathrm{ClpB}$ in bacteria) in some organisms or acting alone in others (e.g. in metazoans). The very first connection between the latter scenario and bacterial sHsps was provided by Veinger and colleagues (Veinger et al., 1998), who in vitro explored disaggregation and refolding mediated by E. coli DnaK-ClpB bi-chaperone system in the presence or absence of $\mathrm{IbpB}$ (Figure 2). While the ability of sHsps to form assemblies was already known, the authors aimed to investigate whether IbpB binding has an impact on substrate disaggregation. They showed that IbpB does indeed influence the later refolding, facilitating it when present upon the denaturation step. Together with similar studies on an eukaryotic sHsp (Ehrnsperger et al., 1997), it seeded the hypothesis, that sHsps stabilize folding intermediates into assemblies that constitute a reservoir for their subsequent refolding. Later, yet another link between sHsps and 'big' chaperones was provided by Mogk et al. (2003b). They showed that E. coli sHsps, IbpA and IbpB, cooperate with $\mathrm{ClpB}$ and the DnaK system in vitro and in vivo and that IbpA and IbpB become essential for cell viability when DnaK levels are reduced (Mogk et al., 2003a).

Intuitively, one could think that such a refolding reservoir - as it was shown for E. coli IbpB (Veinger et al., 1998) - should generally facilitate disaggregation by $\mathrm{Hsp} 70-\mathrm{Hsp} 100$ bichaperone system. Indeed, it was reported that the denaturation of several different substrates in the presence of sHsps substantially increases the subsequent ClpB-DnaKdependent refolding efficiency (Matuszewska et al., 2005; Mogk et al., 2003a; Mogk et al., 2003b). However, it was also noticed that it is not universal. E. coli IbpA protein, which efficiently forms assemblies, was reported to possess an evident inhibitory activity towards disaggregation and refolding in the absence of its IbpB paralog (Matuszewska et al., 2005; Ratajczak et al., 2009). This Janus-faced behavior of sHsps was not really explained until 2017, when Zwirowski and colleagues (Zwirowski et al., 2017) proposed a model for the interplay between sHsps and Hsp70 system in the refolding of aggregated substrates. They showed that the sHsp-induced inhibition is observed solely at low Hsp70 concentration and above a certain Hsp70 threshold sHsp presence in aggregates provides a substantial boost in disaggregation. This led to several mechanistic experiments, where the authors showed that the longpursued sHsp interaction with other chaperones is, in fact, indirect. It is based on a simple Hsp70-sHsp competition for substrate polypeptides. Only the Hsp70 molecules that win the competition and bind the aggregate may further recruit, dock and stimulate the Hsp100 disaggregase for a polypeptide extraction (Rosenzweig et al., 2013; Miot et al., 2011; Liberek et al., 2008; Mogk et al., 2015) (Figure 2). The Hsp70-dependent release of sHsp from aggregates formed in stressed cells was also previously shown in cyanobacteria (Basha et al., 2004).

Given that sHsps have to effectively bind misfolding peptides and swiftly release them upon the Hsp70 action, a serious evolutionary trade-off has emerged. One sHsp simply cannot be a stable binder to form assemblies and, at the same time, promote disaggregation. Although most bacteria utilize just one sHsp, there are species expressing more of them (Haslbeck et al., 2005). Recently, Obuchowski et al. (2019) have shown that species from Enterobacterales clade have evolved an sHsp system of two cooperating components. One is a canonical IbpA that is a tight binder that is hard to outcompete from the substrate by Hsp70, and the other one, IbpB, is unable to stably bind the substrate and, therefore, can hardly modulate polypeptide aggregation (Ratajczak et al., 2009; Obuchowski et al., 2019). Such observations about the properties of these two sHsps 
come not only from in vitro experiments but also from in vivo studies which showed that IbpA is present exclusively in the aggregated protein fraction, while IbpB in the absence of IbpA is found mostly in the cytosolic soluble fraction (Kuczynska-Wisnik et al., 2002). Acting as a complex, they can both efficiently scavenge unfolding polypeptides and be removed from assemblies upon Hsp70 binding (Obuchowski et al., 2019). However, it is worth noting that using this data to induce conclusions about non-Enterobacterales should be done with great care, as it would require an assumption of convergence. It was already shown not to be the case for D. radiodurans, also expressing two paralogous sHsps that do not cooperate with each other, at least not in counteracting aggregation (Bepperling et al., 2012).

As already noted, different bacteria may contain varying numbers of sHsps. Known examples include species with only single sHsp, such as Erwinia amylovora or Vibrio harveyi (Klein et al., 2001; Obuchowski et al., 2019), two sHsps, like E. coli or D. radiodurans (Bepperling et al., 2012; Obuchowski et al., 2019) as well as three sHsps, like L. plantarum (Arena et al., 2019) or Pseudomonas putida (Krajewski et al., 2014). There are also more extreme cases like rhizobia, which possess large superfamily of sHsp, grouped in two distinct classes. The best studied example, Bradyrhizobium japonicum, contains seven identified sHsp genes as well as at least five more sHsps indicated by proteomic analysis (Münchbach et al., 1999). Studied examples of sHsps from bacteria expressing single and multiple sHsps revealed that their general principles of function are somewhat similar to sHsps from E. coli. sHsps from both single (Klein et al., 2001; Obuchowski et al., 2019) and multi-protein sHsp systems (Studer and Narberhaus 2000; Krajewski et al., 2014) form potentially mixed (in case of multi-protein systems) oligomers and interact with substrate proteins when the temperature rises (Studer and Narberhaus 2000; Klein et al., 2001; Obuchowski et al., 2019). Still, to date knowledge on bacterial sHsps would benefit from in depth analysis of sHsp-substrates complexes, both in terms of formation kinetics and structural organization. The spectrum of

\section{REFERENCES}

Allen, S. P., Polazzi, J. O., Gierse, J. K., and Easton, A. M. (1992). Two Novel Heat Shock Genes Encoding Proteins Produced in Response to Heterologous Protein Expression in Escherichia coli. J. Bacteriol. 174 (21), 6938-6947. doi:10.1128/jb. 174.21.6938-6947.1992 Available at: http://www.ncbi.nlm.nih.gov/pubmed/ 1356969

Altenhoff, A. M., Gil, M., Gonnet, G. H., and Dessimoz, C. (2013). Inferring Hierarchical Orthologous Groups from Orthologous Gene Pairs. PLoS One 8 (1), e53786. doi:10.1371/journal.pone.0053786 Available at: https://www.ncbi. nlm.nih.gov/pubmed/23342000

Arena, M. P., Capozzi, V., Longo, A., Russo, P., Weidmann, S., Rieu, A., et al. (2019). The Phenotypic Analysis of Lactobacillus Plantarum Shsp Mutants Reveals a Potential Role for Hsp1 in Cryotolerance. Front. Microbiol. 10 (838). doi:10.3389/fmicb.2019.00838 Available at: https://www.frontiersin.org/article/ 10.3389/fmicb.2019.00838

Basha, E., Jones, C., Blackwell, A. E., Cheng, G., Waters, E. R., Samsel, K. A., et al. (2013). An Unusual Dimeric Small Heat Shock Protein Provides Insight into the Mechanism of This Class of Chaperones. J. Mol. Biol. 425 (10), 1683-1696.
sHsps protein substrates at stress conditions is also hardly defined.

\section{CONCLUSION}

Summing up, although different bacteria possess a different number of sHsp genes of limited conservation, all bacterial sHsps have consensus features defining their general activity. Most of all, it is the ATP-independent ability to bind the substrate following heat activation. sHsps bind substrates either stably, storing polypeptides for subsequent Hsp100-Hsp70 disaggregating machinery action, or transiently for unfolding prevention. Both activities positively influence protein homeostasis, increasing bacterial capabilities to survive stress conditions. These activities are always orchestrated by the very same, strikingly conserved structure of a-crystallin domain and flanking termini - showing that for this purpose it is a highly optimal solution that was provided very early in the evolution of chaperone systems.

\section{AUTHOR CONTRIBUTIONS}

All authors listed have made a substantial, direct, and intellectual contribution to the work and approved it for publication.

\section{FUNDING}

This work was funded by a grant of the Polish National Science Centre (OPUS 17 2019/33/B/NZ1/00352). IO is a recipient of START program (63.2020) from the Foundation for Polish Science.

\section{ACKNOWLEDGMENTS}

We thank Dagmara Mróz and Hubert Wyszkowski for discussions and critical reading of the manuscript.

doi:10.1016/j.jmb.2013.02.011 Available at: http://www.ncbi.nlm.nih.gov/ pubmed $/ 23416558$

Basha, E., Lee, G. J., Breci, L. A., Hausrath, A. C., Buan, N. R., Giese, K. C., et al. (2004). The Identity of Proteins Associated with a Small Heat Shock Protein during Heat Stress In Vivo Indicates that These Chaperones Protect a Wide Range of Cellular Functions. J. Biol. Chem. 279 (9), 7566-7575. doi:10.1074/jbc. m310684200 Available at: https://www.ncbi.nlm.nih.gov/pubmed/14662763

Benesch, J. L. P., Aquilina, J. A., Baldwin, A. J., Rekas, A., Stengel, F., Lindner, R. A., et al. (2010). The Quaternary Organization and Dynamics of the Molecular Chaperone HSP26 Are Thermally Regulated. Chem. Biol. 17 (9), 1008-1017. doi:10.1016/j.chembiol.2010.06.016

Bepperling, A., Alte, F., Kriehuber, T., Braun, N., Weinkauf, S., Groll, M., et al. (2012). Alternative Bacterial Two-Component Small Heat Shock Protein Systems. Proc. Natl. Acad. Sci. 109 (50), 20407-20412. doi:10.1073/pnas. 1209565109

Bissonnette, S. A., Rivera-Rivera, I., Sauer, R. T., and Baker, T. A. (2010). The IbpA and IbpB Small Heat-Shock Proteins Are Substrates of the AAA+ Lon Protease. Mol. Microbiol. 75 (6), 1539-1549. doi:10.1111/j.1365-2958.2010.07070.x

Capozzi, V., Weidmann, S., Fiocco, D., Rieu, A., Hols, P., Guzzo, J., et al. (2011). Inactivation of a Small Heat Shock Protein Affects Cell Morphology and 
Membrane Fluidity in Lactobacillus Plantarum WCFS1. Res. Microbiol. 162 (4), 419-425. doi:10.1016/j.resmic.2011.02.010

Chang, Z., Primm, T. P., Jakana, J., Lee, I. H., Serysheva, I., Chiu, W., et al. (1996). Mycobacterium tuberculosis 16-kDa Antigen (Hsp16.3) Functions as an Oligomeric Structure In Vitro to Suppress Thermal Aggregation. J. Biol. Chem. 271 (12), 7218-7223. doi:10.1074/jbc.271.12.7218 Available at: https://www.ncbi.nlm.nih.gov/pubmed/8636160

Chernova, L. S., Bogachev, M. I., Chasov, V. V., Vishnyakov, I. E., and Kayumov, A. R. (2020). N- and C-Terminal Regions of the Small Heat Shock Protein IbpA from Acholeplasma Laidlawii Competitively Govern its Oligomerization Pattern and Chaperone-like Activity. RSC Adv. 10 (14), 8364-8376. doi:10. 1039/c9ra10172a

Chuang, S. E., and Blattner, F. R. (1993). Characterization of Twenty-Six New Heat Shock Genes of Escherichia coli. J. Bacteriol. 175 (16), 5242-5252. doi:10.1128/ jb.175.16.5242-5252.1993 Available at: https://jb.asm.org/content/jb/175/16/ 5242.full.pdf

Ehrnsperger, M., Graber, S., Gaestel, M., and Buchner, J. (1997). Binding of Nonnative Protein to Hsp25 during Heat Shock Creates a Reservoir of Folding Intermediates for Reactivation. EMBO J. 16 (2), 221-229. doi:10.1093/emboj/ 16.2.221 Available at: http://www.ncbi.nlm.nih.gov/pubmed/9029143.

Friedrich, K. L., Giese, K. C., Buan, N. R., and Vierling, E. (2004). Interactions between Small Heat Shock Protein Subunits and Substrate in Small Heat Shock Protein-Substrate Complexes. J. Biol. Chem. 279 (2), 1080-1089. doi:10.1074/ jbc.m311104200

Fu, X., Shi, X., Yin, L., Liu, J., Joo, K., Lee, J., et al. (2013). Small Heat Shock Protein IbpB Acts as a Robust Chaperone in Living Cells by Hierarchically Activating its Multi-type Substrate-Binding Residues. J. Biol. Chem. 288 (17), 11897-11906. doi:10.1074/jbc.m113.450437 Available at: http://www.ncbi.nlm.nih.gov/ pubmed $/ 23486475$

Fu, X., Zhang, H., Zhang, X., Cao, Y., Jiao, W., Liu, C., et al. (2005). A Dual Role for the N-Terminal Region of Mycobacterium tuberculosis Hsp16.3 in SelfOligomerization and Binding Denaturing Substrate Proteins. J. Biol. Chem. 280 (8), 6337-6348. doi:10.1074/jbc.m406319200

Gaubig, L. C., Waldminghaus, T., and Narberhaus, F. (2011). Multiple Layers of Control Govern Expression of the Escherichia coli ibpAB Heat-Shock Operon. Microbiology (Reading) 157 (Pt 1), 66-76. doi:10.1099/mic.0.043802-0 Available at: https://www.ncbi.nlm.nih.gov/pubmed/20864473

Haslbeck, M., Franzmann, T., Weinfurtner, D., and Buchner, J. (2005). Some like it Hot: the Structure and Function of Small Heat-Shock Proteins. Nat. Struct. Mol. Biol. 12 (10), 842-846. doi:10.1038/nsmb993 Available at: http://www.ncbi.nlm. nih.gov/pubmed/16205709

Haslbeck, M., and Vierling, E. (2015). A First Line of Stress Defense: Small Heat Shock Proteins and Their Function in Protein Homeostasis. J. Mol. Biol. 427 (7), 1537-1548. doi:10.1016/j.jmb.2015.02.002 Available at: http://www.ncbi.nlm. nih.gov/pubmed/25681016

Haslbeck, M., Weinkauf, S., and Buchner, J. (2019). Small Heat Shock Proteins: Simplicity Meets Complexity. J. Biol. Chem. 294 (6), 2121-2132. doi:10.1074/jbc. rev118.002809 Available at: http://www.jbc.org/content/294/6/2121.abstract

Hilario, E., Martin, F. J. M., Bertolini, M. C., and Fan, L. (2011). Crystal Structures of Xanthomonas Small Heat Shock Protein Provide a Structural Basis for an Active Molecular Chaperone Oligomer. J. Mol. Biol. 408 (1), 74-86. doi:10. 1016/j.jmb.2011.02.004

Horvath, I., Glatz, A., Varvasovszki, V., Torok, Z., Pali, T., Balogh, G., et al. (1998). Membrane Physical State Controls the Signaling Mechanism of the Heat Shock Response in Synechocystis PCC 6803: Identification of Hsp17 as a "fluidity Gene". Proc. Natl. Acad. Sci. 95 (7), 3513-3518. doi:10.1073/pnas.95. 7.3513

Hu, Y., Movahedzadeh, F., Stoker, N. G., and Coates, A. R. M. (2006). Deletion of the Mycobacterium tuberculosis a-Crystallin-Like hspX Gene Causes Increased Bacterial Growth In Vivo. Iai 74 (2), 861-868. doi:10.1128/iai.74.2.861-868. 2006 Available at: https://www.ncbi.nlm.nih.gov/pubmed/16428728

Jiao, W., Qian, M., Li, P., Zhao, L., and Chang, Z. (2005). The Essential Role of the Flexible Termini in the Temperature-Responsiveness of the Oligomeric State and Chaperone-like Activity for the Polydisperse Small Heat Shock Protein IbpB from Escherichia coli. J. Mol. Biol. 347 (4), 871-884. doi:10.1016/j.jmb. 2005.01.029

Kennaway, C. K., Benesch, J. L. P., Gohlke, U., Wang, L., Robinson, C. V., Orlova, E. V., et al. (2005). Dodecameric Structure of the Small Heat Shock Protein Acr1 from Mycobacterium tuberculosis. J. Biol. Chem. 280 (39), 33419-33425. doi:10. 1074/jbc.m504263200

Kitagawa, M., Miyakawa, M., Matsumura, Y., and Tsuchido, T. (2002). Escherichia coli Small Heat Shock Proteins, IbpA and IbpB, Protect Enzymes from Inactivation by Heat and Oxidants. Eur. J. Biochem. 269 (12), 2907-2917. doi:10.1046/j.1432-1033.2002.02958.x

Klein, G., Laskowska, E., Taylor, A., and Lipińska, B. (2001). IbpA/B Small HeatShock Protein of Marine Bacterium Vibrio Harveyi Binds to Proteins Aggregated in a Cell during Heat Shock. Mar. Biotechnol. 3 (4), 346-354. doi:10.1007/s10126001-0009-2

Krajewski, S. S., Joswig, M., Nagel, M., and Narberhaus, F. (2014). A Tricistronic Heat Shock Operon Is Important for Stress Tolerance ofPseudomonas Putidaand Conserved in Many Environmental Bacteria. Environ. Microbiol. 16 (6), 1835-1853. doi:10.1111/1462-2920.12432 Available at: https:// sfamjournals.onlinelibrary.wiley.com/

Kriehuber, T., Rattei, T., Weinmaier, T., Bepperling, A., Haslbeck, M., and Buchner, J. (2010). Independent Evolution of the Core Domain and its Flanking Sequences in Small Heat Shock Proteins. FASEB j. 24 (10), 3633-3642. doi:10.1096/fj.10-156992

Kuczynska-Wisnik, D., Kedzierska, S., Matuszewska, E., Lund, P., Taylor, A., Lipinska, B., et al. (2002). The Escherichia coli Small Heat-Shock Proteins IbpA and IbpB Prevent the Aggregation of Endogenous Proteins Denatured In Vivo during Extreme Heat Shock. Microbiology 148 (Pt 6), 1757-1765. doi:10.1099/ 00221287-148-6-1757 Available at: http://www.ncbi.nlm.nih.gov/pubmed/ 12055295

Kuczyńska-Wisńik, D., Laskowska, E., and Taylor, A. (2001). Transcription of the ibpB Heat-Shock Gene Is under Control of Sigma(32)- and Sigma(54)Promoters, a Third Regulon of Heat-Shock Response. Biochem. Biophys. Res. Commun. 284 (1), 57-64. doi:10.1006/bbrc.2001.4926

Laskowska, E., Wawrzynów, A., and Taylor, A. (1996). IbpA and IbpB, the New Heat-Shock Proteins, Bind to Endogenous Escherichia coli Proteins Aggregated Intracellularly by Heat Shock. Biochimie 78 (2), 117-122. doi:10.1016/03009084(96)82643-5

Liberek, K., Lewandowska, A., and Ziętkiewicz, S. (2008). Chaperones in Control of Protein Disaggregation. Embo J. 27 (2), 328-335. doi:10.1038/sj.emboj.7601970

Maitre, M., Weidmann, S., Dubois-Brissonnet, F., David, V., Covès, J., and Guzzo, J. (2014). Adaptation of the Wine Bacterium Oenococcus Oeni to Ethanol Stress: Role of the Small Heat Shock Protein Lo18 in Membrane Integrity. Appl. Environ. Microbiol. 80 (10), 2973-2980. doi:10.1128/aem.04178-13

Maitre, M., Weidmann, S., Rieu, A., Fenel, D., Schoehn, G., Ebel, C., et al. (2012). The Oligomer Plasticity of the Small Heat-Shock Protein Lo18 from Oenococcus Oeni Influences its Role in Both Membrane Stabilization and Protein Protection. Biochem. J. 444, 97-104. doi:10.1042/bj20120066

Mani, N., Bhandari, S., Moreno, R., Hu, L., Prasad, B. V. V., and Suguna, K. (2016). Multiple Oligomeric Structures of a Bacterial Small Heat Shock Protein. Sci. Rep. 6, 24019. doi:10.1038/srep24019 Available at: https://www.ncbi.nlm.nih. gov/pubmed/27053150

Matuszewska, M., Kuczyńska-Wiśnik, D., Laskowska, E., and Liberek, K. (2005). The Small Heat Shock Protein IbpA of Escherichia coli Cooperates with IbpB in Stabilization of Thermally Aggregated Proteins in a Disaggregation Competent State. J. Biol. Chem. 280 (13), 12292-12298. doi:10.1074/jbc.m412706200 Available at: http://www.ncbi.nlm.nih.gov/pubmed/15665332

Miot, M., Reidy, M., Doyle, S. M., Hoskins, J. R., Johnston, D. M., Genest, O., et al. (2011). Species-specific Collaboration of Heat Shock Proteins (Hsp) 70 and 100 in Thermotolerance and Protein Disaggregation. Proc. Natl. Acad. Sci. 108 (17), 6915-6920. doi:10.1073/pnas.1102828108 Available at: http://www.ncbi.nlm. nih.gov/pubmed/21474779

Miwa, T., Chadani, Y., and Taguchi, H. (2021). Escherichia coli Small Heat Shock Protein IbpA Is an Aggregation-sensor that Self-regulates its Own Expression at Posttranscriptional Levels. Mol. Microbiol. 115 (1), 142-156. doi:10.1111/mmi. 14606 Available at: https://www.ncbi.nlm.nih.gov/pubmed/32959419

Mogk, A., Deuerling, E., Vorderwülbecke, S., Vierling, E., and Bukau, B. (2003a). Small Heat Shock Proteins, ClpB and the DnaK System Form a Functional Triade in Reversing Protein Aggregation. Mol. Microbiol. 50 (2), 585-595. doi:10.1046/j.1365-2958.2003.03710.x Available at: http://www.ncbi.nlm.nih. gov/pubmed/14617181

Mogk, A., Schlieker, C., Friedrich, K. L., Schönfeld, H.-J., Vierling, E., and Bukau, B. (2003b). Refolding of Substrates Bound to Small Hsps Relies on a 
Disaggregation Reaction Mediated Most Efficiently by ClpB/DnaK. J. Biol. Chem. 278 (33), 31033-31042. doi:10.1074/jbc.m303587200 Available at: http:// www.ncbi.nlm.nih.gov/pubmed/12788951

Mogk, A., Tomoyasu, T., Goloubinoff, P., Rudiger, S., Roder, D., Langen, H., et al. (1999). Identification of Thermolabile Escherichia coli Proteins: Prevention and Reversion of Aggregation by DnaK and ClpB. EMBO J. 18 (24), 6934-6949. doi:10.1093/emboj/18.24.6934 Available at: http://www.ncbi.nlm.nih.gov/ pubmed/10601016

Mogk, Axel., Kummer, Eva., and Bukau, Bernd. (2015). Cooperation of Hsp70 and Hsp100 Chaperone Machines in Protein Disaggregation. Front. Mol. Biosciences 2 (22). doi:10.3389/fmolb.2015.00022 Available at: https://www. frontiersin.org/article/10.3389/fmolb.2015.00022

Münchbach, M., Nocker, A., and Narberhaus, F. (1999). Multiple Small Heat Shock Proteins in Rhizobia. J. Bacteriol. 181 (1), 83-90.

Nakamoto, H., and Honma, D. (2006). Interaction of a Small Heat Shock Protein with Light-Harvesting Cyanobacterial Phycocyanins under Stress Conditions. Febs Lett. 580 (13), 3029-3034. doi:10.1016/j.febslet.2006.04.047

Narberhaus, F., Waldminghaus, T., and Chowdhury, S. (2006). RNA Thermometers. FEMS Microbiol. Rev. 30 (1), 3-16. doi:10.1111/j.1574-6976. 2005.004.x

Obuchowski, I., Pirog, A., Stolarska, M., Tomiczek, B., and Liberek, K. (2019). Duplicate Divergence of Two Bacterial Small Heat Shock Proteins Reduces the Demand for Hsp70 in Refolding of Substrates. Plos Genet. 15 (10), e1008479. doi:10.1371/journal.pgen.1008479 Available at: https://www.ncbi.nlm.nih.gov/ pubmed/31652260

Painter, A. J., Jaya, N., Basha, E., Vierling, E., Robinson, C. V., and Benesch, J. L. P. (2008). Real-time Monitoring of Protein Complexes Reveals Their Quaternary Organization and Dynamics. Chem. Biol. 15 (3), 246-253. doi:10.1016/j. chembiol.2008.01.009

Ratajczak, E., Stróżecka, J., Matuszewska, M., Ziętkiewicz, S., Kuczyńska-Wiśnik, D., Laskowska, E., et al. (2010). IbpA the Small Heat Shock Protein fromEscherichia Coliforms Fibrils in the Absence of its Cochaperone IbpB. FEBS Lett. 584 (11), 2253-2257. doi:10.1016/j.febslet.2010.04.060 Available at: https://www.ncbi.nlm.nih.gov/pubmed/20433838

Ratajczak, E., Ziętkiewicz, S., and Liberek, K. (2009). Distinct Activities of Escherichia coli Small Heat Shock Proteins IbpA and IbpB Promote Efficient Protein Disaggregation. J. Mol. Biol. 386 (1), 178-189. doi:10.1016/j.jmb.2008. 12.009 Available at: http://www.ncbi.nlm.nih.gov/pubmed/19101567

Richmond, C., Glasner, J. D., Mau, R., Jin, H., and Blattner, F. R. (1999). Genomewide Expression Profiling in Escherichia coli K-12. Nucleic Acids Res. 27 (19), 3821-3835. doi:10.1093/nar/27.19.3821 Available at: https://www.ncbi.nlm.nih. gov/pubmed/10481021

Rosenzweig, R., Moradi, S., Zarrine-Afsar, A., Glover, J. R., and Kay, L. E. (2013). Unraveling the Mechanism of Protein Disaggregation through a ClpB-DnaK Interaction. Science 339 (6123), 1080-1083. doi:10.1126/ science. 1233066

Shearstone, J. R., and Baneyx, F. (1999). Biochemical Characterization of the Small Heat Shock Protein IbpB from Escherichia coli. J. Biol. Chem. 274 (15),
9937-9945. doi:10.1074/jbc.274.15.9937 Available at: https://www.ncbi.nlm. nih.gov/pubmed/10187768

Strózecka, J., Chrusciel, E., Górna, E., Szymanska, A., Ziętkiewicz, S., and Liberek, K. (2012). Importance of N- and C-Terminal Regions of IbpA, Escherichia coli Small Heat Shock Protein, for Chaperone Function and Oligomerization. J. Biol. Chem. 287 (4), 2843-2853. doi:10.1074/jbc.M111.273847 Available at: http:// www.ncbi.nlm.nih.gov/pubmed/22139842

Studer, S., and Narberhaus, F. (2000). Chaperone Activity and Homo- and HeteroOligomer Formation of Bacterial Small Heat Shock Proteins. J. Biol. Chem. 275 (47), 37212-37218. doi:10.1074/jbc.m004701200 Available at: https://www. sciencedirect.com/science/article/pii/S0021925820886372

Torok, Z., Goloubinoff, P., Horvath, I., Tsvetkova, N. M., Glatz, A., Balogh, G., et al. (2001). Synechocystis HSP17 Is an Amphitropic Protein that Stabilizes HeatStressed Membranes and Binds Denatured Proteins for Subsequent ChaperoneMediated Refolding. Proc. Natl. Acad. Sci. 98 (6), 3098-3103. doi:10.1073/pnas. 051619498

Ungelenk, S., Moayed, F., Ho, C. T., Grousl, T., Scharf, A., Mashaghi, A., et al. (2016). .Small Heat Shock Proteins Sequester Misfolding Proteins in NearNative Conformation for Cellular Protection and Efficient Refolding, Nat. Commun. 7 (13673). doi:10.1038/ncomms1367331

Valdez-Cruz, N. A., Ramírez, O. T., and Trujillo-Roldán, M. A. (2011). Molecular Responses ofE. Colicaused by Heat Stress and Recombinant Protein Production during Temperature Induction. Bioengineered Bugs 2 (2), 105-110. doi:10.4161/bbug.2.2.14316 Available at: https://www.ncbi. nlm.nih.gov/pubmed/21636998

Veinger, L., Diamant, S., Buchner, J., and Goloubinoff, P. (1998). The Small HeatShock Protein IbpB from Escherichia coli Stabilizes Stress-Denatured Proteins for Subsequent Refolding by a Multichaperone Network. J. Biol. Chem. 273 (18), 11032-11037. doi:10.1074/jbc.273.18.11032

Waldminghaus, T., Gaubig, L. C., Klinkert, B., and Narberhaus, F. (2009). TheEscherichia coliibpA Thermometer Is Comprised of Stable and Unstable Structural Elements. RNA Biol. 6 (4), 455-463. doi:10.4161/rna.6.4.9014 Available at: https://www.ncbi.nlm.nih.gov/pubmed/19535917

Zwirowski, S., Klosowska, A., Obuchowski, I., Nillegoda, N. B., Pirog, A., Zieztkiewicz, S., et al. (2017). Hsp70 Displaces Small Heat Shock Proteins from Aggregates to Initiate Protein Refolding. Embo J. 36 (6), 783-796.

Conflict of Interest: The authors declare that the research was conducted in the absence of any commercial or financial relationships that could be construed as a potential conflict of interest.

Copyright $\odot 2021$ Obuchowski, Karaś and Liberek. This is an open-access article distributed under the terms of the Creative Commons Attribution License (CC BY). The use, distribution or reproduction in other forums is permitted, provided the original author(s) and the copyright owner(s) are credited and that the original publication in this journal is cited, in accordance with accepted academic practice. No use, distribution or reproduction is permitted which does not comply with these terms. 